\title{
Empathic Interactions in Automated Vehicles \#EmpathicCHI
}

\author{
Karl Daher \\ karl.daher@hes-so.ch \\ HES-SO University of Applied \\ Sciences and Arts Western \\ Switzerland \\ Fribourg, Switzerland \\ Jacky Casas \\ jacky.casas@hes-so.ch \\ HES-SO University of Applied \\ Sciences and Arts Western \\ Switzerland \\ Fribourg, Switzerland \\ Abdallah El Ali \\ Abdallah.El.Ali@cwi.nl \\ Distributed \& Interactive Systems \\ group, Centrum Wiskunde \& \\ Informatica (CWI) \\ Amsterdam, Netherlands
}

\author{
Marine Capallera \\ marine.capallera@hes-so.ch \\ HES-SO University of Applied \\ Sciences and Arts Western \\ Switzerland \\ Fribourg, Switzerland \\ Quentin Meteier \\ quentin.meteier@hes-so.ch \\ HES-SO University of Applied \\ Sciences and Arts Western \\ Switzerland \\ Fribourg, Switzerland
}

\author{
Giorgio Mario Grasso \\ giorgiomario.grasso@unime.it \\ University of Messina \\ Messina, Italy
}

\author{
Chiara Lucifora \\ chiara.lucifora@unime.it \\ University of Messina \\ Messina, Italy \\ Mira El Kamali \\ mira.elkamali@hes-so.ch \\ HES-SO University of Applied \\ Sciences and Arts Western \\ Switzerland \\ Fribourg, Switzerland \\ Gérard Chollet \\ gerard.chollet@telecom-sudparis.eu \\ CNRS-SAMOVAR \\ Paris, France
}

\author{
Omar Abou Khaled \\ omar.aboukhaled@hes-so.ch \\ HES-SO University of Applied \\ Sciences and Arts Western \\ Switzerland \\ Fribourg, Switzerland
}

\author{
Elena Mugellini \\ elena.mugellini@hes-so.ch \\ HES-SO University of Applied \\ Sciences and Arts Western \\ Switzerland \\ Fribourg, Switzerland
}

\begin{abstract}
Automation in driving will change the role of the drivers from actor to passive supervisor. Although the vehicle will be responsible for driving manoeuvres, drivers will need to rely on automation and understand its decisions to establish a trusting relationship between them and the vehicle. Progress has been made in conversational agents and affective machines recently. Moreover, it seems to be promising in this establishment of trust between humans and machines. We believe it is essential to investigate the use of emotional conversational agents in the automotive context to build a solid relationship between the driver and the vehicle. In this workshop, we aim at gathering researchers and industry practitioners from different fields of $\mathrm{HCI}, \mathrm{ML} / \mathrm{AI}, \mathrm{NLU}$ and psychology to brainstorm about affective machines, empathy and conversational agent with a particular focus on human-vehicle interaction. Questions like "What would be the specificities of a multimodal and empathic agent in a car?", "How the agent could make the driver aware of the

Permission to make digital or hard copies of part or all of this work for personal or classroom use is granted without fee provided that copies are not made or distributed for profit or commercial advantage and that copies bear this notice and the full citation on the first page. Copyrights for third-party components of this work must be honored.

For all other uses, contact the owner/author(s).

CHI '21 Extended Abstracts, May 8-13, 2021, Yokohama, Japan

(c) 2021 Copyright held by the owner/author(s)

ACM ISBN 978-1-4503-8095-9/21/05.

https://doi.org/10.1145/3411763.3441359
\end{abstract}

situation?" and "How to measure the trust between the user and the autonomous vehicle?" will be addressed in this workshop.

\section{CCS CONCEPTS}

- Human-centered computing $\rightarrow$ Human computer interaction (HCI).

\section{KEYWORDS}

HCI, Empathy, Human Vehicle Interaction, Multimodal Interaction, Conversational Agent

ACM Reference Format:

Karl Daher, Marine Capallera, Chiara Lucifora, Jacky Casas, Quentin Meteier, Mira El Kamali, Abdallah El Ali, Giorgio Mario Grasso, Gérard Chollet, Omar Abou Khaled, and Elena Mugellini. 2021. Empathic Interactions in Automated Vehicles \#EmpathicCHI. In CHI Conference on Human Factors in Computing Systems Extended Abstracts (CHI '21 Extended Abstracts), May 8-13, 2021, Yokohama, Japan. ACM, New York, NY, USA, 4 pages. https://doi.org/10.1145/3411763.3441359

\section{BACKGROUND}

Drivers are often held responsible for road accidents [7]. Various factors such as fatigue, stress or distraction can affect the driver's performance and lead to accidents. To solve that problem, car manufacturers aim at supporting the driver by increasing the level of 
automation in cars. The Society of Automotive Engineers (SAE) developed a taxonomy to define the levels of automation in cars [3]. In the next decades, we may observe the emergence of highly (Level 4) or fully automated driving (Level 5). From level 4 and more, the car will be in charge of all decisions, with regard to driving manoeuvres as well as ethical choices. To convey its decisions and choices, various in-car interfaces involving different modalities can be investigated. It includes visual modality using screens (dashboard, central console or head-up display) or lights, but also sounds or vibrations. Concepts of multimodal interaction could also be designed to convey information through different interaction modalities. If we want that drivers accept to trust such systems and follow car's decisions, it might be comforting for drivers to explicit vehicle's decisions with empathy [11].

An empathetic relation between a machine and a human has shown an effect on conversations to be unstrung and more spontaneous. It can also increase the user engagement and trust towards the machine $[9,10]$ and allows the user to better cope with stress and frustration [6]. It has also shown a positive effect on task outcome. In certain specific domains such as health or client relations, empathy is privileged in the relationship with other individuals [8]. Therefore, bringing empathy in an automated car might show similar effects on the driver. In the context of automated driving, the main issue is to help improve drivers' trust and acceptance by building empathy through multimodal interaction with the driver.

The idea of this workshop is to create a new design of empathic multimodal conversational agent (CA) to solve predefined use case scenarios. The goal is to define the best multimodal form and empathic capability of the conversational agent to increase drivers' trust and Situation Awareness (SA) during automated driving. A second part of the participants' reflection will be focused on selecting measures to evaluate the driver's state to ensure that drivers will feel better and trust these systems.

\section{ORGANIZERS}

The organizing team consists of academic researchers and practitioners in the field of HCI, ML/AI, NLU, autonomous vehicles, and psychology. Our organizers have been actively researching the topic of human-vehicles interactions, empathic HCI and AI conversational agents. Some of our team members have organized workshops on ubiquitous chatbots interactions, multimodal e-coaches interactions and explainable $\mathrm{AI}$ in autonomous vehicles at UBICOMP/ISWC [1], ICMI [2], and AutomotiveUI [5].

The cross-disciplinary nature of our team will allow the organizers to attract workshop attendees from diverse backgrounds, stimulate interdisciplinary discussion during the workshop, and disseminate the results in each of their communities.

Karl Daher is a $\mathrm{PhD}$ at the University of Fribourg in collaboration with the HumanTech institute from the University of Applied Sciences and Arts Western Switzerland. Co-creator of Empathic Labs group. Karl's main interest is empathy in human-computer interaction and the application of empathy in real-world scenarios to improve humans mental and physical well-being.

Marine Capallera is a $\mathrm{PhD}$ student at University of Fribourg and the HES-SO University of Applied Sciences and Arts Western Switzerland. She is working on conditionally automated driving and she is focusing more specifically on multimodal Human-Vehicle Interaction model for supervision.

Chiara Lucifora is a PhD Student at the University of Messina, Department of Cognitive Science. Her research interest concerns the study of moral psychology in the field of artificial intelligence, focuses on the topic of Autonomous Driving System.

Jacky Casas is a PhD student at the University of Fribourg and the HES-SO University of Applied Sciences and Arts Western Switzerland. His research interests are related to AI, NLP, chatbots and empathic textual interactions. He is the co-founder of Empathic Labs.

Quentin Meteier is a $\mathrm{PhD}$ student at University of Fribourg and the HES-SO University of Applied Sciences and Arts Western Switzerland. He is working on conditionally automated driving and focuses his research on evaluating the physiological state of the driver using machine learning techniques.

Mira El Kamali is a PhD student at the University of Fribourg in collaboration with the Humantech institute at the University of Applied Sciences and Arts Western Switzerland. Her research interest is human-computer interaction and focuses on conversational agents to improve well-being.

Abdallah El Ali is a Tenure-track Researcher at the Distributed \& Interactive Systems group at Centrum Wiskunde \& Informatica (CWI) in the Netherlands. His research focus is on temporal challenges in eliciting, capturing, and predicting human emotions, specifically on usable and effective emotion elicitation and annotation techniques across environments (VR/AR, mobile, wearables, automotive). Website: https://abdoelali.com/

Giorgio Mario Grasso is a Professor at the University of Messina, Department of Cognitive Science, and Director of the NeuroInformatics and Cognitive Science Laboratory. His research fields are Artificial Intelligence, Data Mining, Embedded Computing, Simulation and Cognitive Science.

Gérard Chollet studied Linguistics, Electrical Engineering and Computer Science at the University of California, Santa Barbara where he was granted a $\mathrm{PhD}$ in Computer Science and Linguistics. In 1983, he joined a newly created CNRS research unit at ENST (now Institut Polytechnique de Paris). He supervised more than forty doctoral theses. CNRS decided to grant him an emeritus status in 2012. His main publications are available from http://scholar.google. co.uk/citations? user=NakTCiYAAAAJ\&hl=en.

Omar Abou Khaled is a Professor at the University of Applied Sciences and Arts Western Switzerland. His research fields are Human-Computer Interaction and Wearable and Ubiquitous computing.

Elena Mugellini is a Professor at the University of Applied Sciences and Arts Western Switzerland and head of the HumanTech Institute. Her research fields are Human-Computer Interaction and Data Analytics.

\section{WEBSITE}

Our workshop website (https://sites.google.com/view/empathiccars-workshop) explains the goal and logistics of the workshop, including workshop schedule and organizers' backgrounds. We will post readings related to the workshop theme, updates and news during and after the workshop. 


\section{PRE-WORKSHOP PLANS}

\#EmpathicCHI workshop will recruit participants from inside and outside the $\mathrm{CHI}$ community. The $\mathrm{CHI}$ previous attendees will be invited from the mailing list of $\mathrm{CHI}-\mathrm{ACM}$, the lists of the workshops that we organized previously as well as the mailing lists of groups which we belong to as "Affects, Compagnons Artificiels et Interactions" (ACAI) ${ }^{1}$, the Association Francophone d'Interaction Homme-Machine (AFIHM) ${ }^{2}$, in addition to the meet-ups and on the Wiki-CFP website. Further, we will invite people from outside the academic world to mix with the industry world. The workshop will have a website to present the information, the call for participation and the purpose of the workshop. we will share everything on social media (Instagram, Facebook, Twitter, Medium, LinkedIn). The ideas shared by the participants will derive new concepts and applications related to real-world scenarios.

\section{WORKSHOP STRUCTURE}

The workshop will follow a half-day format (3h40). A preliminary schedule for the workshop is proposed in Table 1 . To start the workshop, an introductory session is given by the organizers to detail the organization and explain the objectives and expected outcomes of this workshop. After that, half of the participants are invited to introduce themselves during a flash presentation pitch session. Attendees have between 2 and 5 minutes each to make a pitch about their background and research interests using only one slide. Another session will take place before the second coffee break. After the first session of flash presentations, an overview of the context will be given by the organizers, including insights and relevant definitions about automated driving as well as multimodal and empathic interaction. After that, some instructions are given to participants about the driving scenarios on which attendees will have to work. Then that, a first session of the workgroup will take place. According to the proposed driving scenario, each should brainstorm about the design of an emphatic human-vehicle multimodal interface (CA \& other interfaces) and the way of measuring emotions, physiological state and trust of the driver. Privacy and ethical aspects related to the collection of biometric data can also be taken into account during the brainstorming. Groups should use the guidelines for automotive conversational user interfaces to structure their ideas [4]. After the brainstorming session, the second flash presentation will occur followed by a short break. Based on the ideas generated from the brainstorming, each group has to create a prototype or a mockup to answer the problem shown by the driving scenario. It is expected that the prototype/mockup gives insights about what information is sent to the driver. The way of conveying that information using empathy and different modalities should also be tackled. To support this phase, online tools for sketching and rapid prototyping will be proposed to support attendees' creativity. Finally, each group has few minutes to present their prototype to the other attendees. All the participants have the opportunity to discuss and ask questions about the prototype designed by each group.

The workshop will be held fully online. Microsoft Teams from our institution (HES-SO) will be used as video-conferencing and

\footnotetext{
${ }^{1}$ https://acai.limsi.fr

${ }^{2}$ https://afihm.org
}

organisational tool. Different channels will be setup on the Microsoft Teams team for each part of the workshop (presentation, brainstorming, creativity session) in order to cluster the discussions. In each channel, files can be shared and video-calls will be made. During the video-calls, participants are encouraged to keep their cameras on to foster interactivity between them. A Miro board will be setup to collaborate in each group for the brainstorming and creativity session. Each group will work on an independent dashboard. Each dashboard will be separated in two parts in order to distinguish the brainstorming and creativity processes. Post-its with interaction modalities and means to measure the state of the driver will be proposed by the organizer in order to facilitate the start of the brainstorming process. A Google Slides deck will be shared among participants in order to gather all the outcome of the creativity session.

\section{POST-WORKSHOP PLANS}

The outcome of the discussions, slides of the presentations and creativity session outcome documents will be made public on the workshop website. The website will serve as a repository of resources for research in the domain of the workshop.

A mailing list will be set up for participants willing to continue collaborating on the topics. This will be a basis for a larger group of people interested in empathic interaction in autonomous vehicles.

\section{CALL FOR PARTICIPATION}

We invite any interested researchers, practitioners and enthusiasts to participate to the CHI 2021 Workshop on "Empathic interactions in automated vehicles \#EmpathicCHI". This one-day online workshop will offer an interdisciplinary forum of discussion for both academics and practitioners interested in setting trust in HumanVehicle interactions by adding empathy with conversational agents.

Relevant topics of the workshop include:

- Interactions models in automated driving

- Concepts of human-vehicle interaction

- Affective conversational agents

- Empathic virtual coach

- Affective computing

- Physiological signal processing

- Virtual agents

- Empathic response generation

- Privacy and ethical aspects according to biometric data collection

Researchers from both academia and industry with interest in empathic interactions between drivers and autonomous vehicles research are invited to attend.

Participants will be invited to present themselves and their current projects related to the workshop topics in one slide. Two sessions are planned during the workshop for these flash presentations.

We invite you to check out our website for important dates, last-minute changes or up to date information. In any case, the organizing team will answer all of your questions by email at empathic.chi21@gmail.com.

$\hookrightarrow$ https://sites.google.com/view/empathic-cars-workshop 


\section{Session}

Introductory session

Flash presentation I (1st half of the participants)

Context presentation : Automated cars, empathy, multimodality, CA

Coffee Break

Instruction and presentation of driving scenario

Brainstorming session (with interventions and activities)

Flash presentation II (2nd half of the participants)

Coffee Break

Creativity session

Presentation and discussion \& Report

Closing session
Duration

$10 \mathrm{~min}$

$30 \mathrm{~min}$

$20 \mathrm{~min}$

$10 \mathrm{~min}$

$10 \mathrm{~min}$

$35 \mathrm{~min}$

$30 \mathrm{~min}$

$10 \mathrm{~min}$

$35 \mathrm{~min}$

$20 \mathrm{~min}$

$10 \mathrm{~min}$

\section{Table 1: Proposed schedule}

\section{REFERENCES}

[1] Leonardo Angelini, Maurizio Caon, Jacky Casas, Federica Cena, Amon Rapp Omar Abou Khaled, and Elena Mugellini. 2018. Ubiquitous Chatbots: Workshop on Wearable and Embodied Conversational Agents. In Proceedings of the 2018 ACM International foint Conference and 2018 International Symposium on Pervasive and Ubiquitous Computing and Wearable Computers (UbiComp '18). Association for Computing Machinery, New York, NY, USA, 1652-1655. https://doi.org/10. $1145 / 3267305.3274146$

[2] Leonardo Angelini, Mira El Kamali, Elena Mugellini, Omar Abou Khaled, Yordan Dimitrov, Vera Veleva, Zlatka Gospodinova, Nadejda Miteva, Richar Wheeler Zoraida Callejas, David Griol, Kawtar Benghazi, Manuel Noguera, Panagiotis Bamidis, Evdokimos Konstantinidis, Despoina Petsani, Andoni Beristain Iraola, Dimitrios I. Fotiadis, Gérard Chollet, Inés Torres, Anna Esposito, and Hannes Schlieter. 2020. First Workshop on Multimodal e-Coaches. In First Workshop on Multimodal e-Coaches (ICMI '20 (TO BE PUBLISHED)). Association for Computing Machinery, Utrecht, Netherlands, 1-3. https://doi.org/10.1145/3382507.3420056

[3] SAE International. 2018. Taxonomy and Definitions for Terms Related to Driving Automation Systems for On-Road Motor Vehicles.

[4] David R. Large, Gary Burnett, and Leigh Clark. 2019. Lessons from Oz: Design Guidelines for Automotive Conversational User Interfaces. In Proceedings of the 11th International Conference on Automotive User Interfaces and Interactive Vehicular Applications: Adjunct Proceedings (Utrecht, Netherlands) (AutomotiveUI '19). Association for Computing Machinery, New York, NY, USA, 335-340. https: //doi.org/10.1145/3349263.3351314

[5] Quentin Meteier, Marine Capallera, Leonardo Angelini, Elena Mugellini, Omar Abou Khaled, Stefano Carrino, Emmanuel De Salis, Stéphane Galland, and Susanne Boll. 2019. Workshop on explainable AI in automated driving: a user-centered interaction approach. In Proceedings of the 11th International Conference on Automotive User Interfaces and Interactive Vehicular Applications: Adjunct Proceedings (AutomotiveUI '19). Association for Computing Machinery, New York, NY, USA, 32-37. https://doi.org/10.1145/3349263.3350762

[6] Hannah Rashkin, Eric Michael Smith, Margaret Li, and Y-Lan Boureau. 2019. Towards Empathetic Open-domain Conversation Models: a New Benchmark and Dataset. arXiv:1811.00207 [cs.CL]

[7] Bryant Walker Smith. 2018. Human error as a cause of vehicle crashes. http: //cyberlaw.stanford.edu/blog/2013/12/human-error-cause-vehicle-crashes

[8] Timo Spring, Jacky Casas, Karl Daher, Elena Mugellini, and Omar Abou Khaled. 2019. Empathic Response Generation in Chatbots. In Proceedings of the 4th Swiss Text analytics Conference (SwissText 2019). CEUR-WS, Winterthur, Switzerland, 10.

[9] Lauren A. Winczewski, Jeffrey D. Bowen, and Nancy L. Collins. 2016. Is Empathic Accuracy Enough to Facilitate Responsive Behavior in Dyadic Interaction? Distinguishing Ability From Motivation. Psychological Science 27, 3 (March 2016), 394-404. https://doi.org/10.1177/0956797615624491

[10] Özge Nilay Yalçın. 2019. Evaluating Empathy in Artificial Agents. In 2019 8th International Conference on Affective Computing and Intelligent Interaction (ACII). IEEE, Cambridge, United Kingdom, 1-7. https://doi.org/10.1109/ACII.2019.8925498 ISSN: 2156-8111.

[11] Sebastian Zepf, Monique Dittrich, Javier Hernandez, and Alexander Schmitt. 2019. Towards Empathetic Car Interfaces: Emotional Triggers While Driving. In Extended Abstracts of the 2019 CHI Conference on Human Factors in Computing Systems (Glasgow, Scotland Uk) (CHI EA '19). Association for Computing Machinery, New York, NY, USA, 1-6. https://doi.org/10.1145/3290607.3312883 